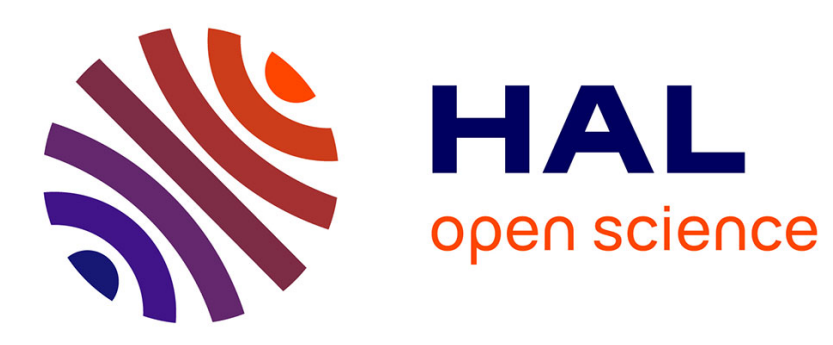

\title{
Exoatmospheric interception problem solved using output feedback law
}

Vincent Andrieu, Hélène Piet-Lahanier

\section{To cite this version:}

Vincent Andrieu, Hélène Piet-Lahanier. Exoatmospheric interception problem solved using output feedback law. Systems and Control Letters, 2006, 55 (8), pp.633-639. 10.1016/j.sysconle.2005.09.017 . hal-00362708

\section{HAL Id: hal-00362708 \\ https://hal.science/hal-00362708}

Submitted on 19 Feb 2009

HAL is a multi-disciplinary open access archive for the deposit and dissemination of scientific research documents, whether they are published or not. The documents may come from teaching and research institutions in France or abroad, or from public or private research centers.
L'archive ouverte pluridisciplinaire HAL, est destinée au dépôt et à la diffusion de documents scientifiques de niveau recherche, publiés ou non, émanant des établissements d'enseignement et de recherche français ou étrangers, des laboratoires publics ou privés. 


\title{
Exoatmospheric interception problem solved using output feedback law
}

\author{
Dr. Vincent Andrieu* and Dr. Hélène Piet Lahanier \\ DPRS, ONERA, BP 72, 29 Ave. de la Division Leclerc, 92322 Châtillon, France
}

\begin{abstract}
Interception problems are often dealt with by separating guidance and autopilot design. Guidance law can be obtained using optimal control theory and autopilot design is performed on a linearized system. In this paper, we introduce a new approach that determines a global guidance and autopilot law, based on direct output feedback design. Application of this method to exoatmospheric interception problem results in good performances. Extension to endoatmospheric case is under investigation.
\end{abstract}

Key words: Guidance, flight control, output feedback.

\section{Introduction}

The problem studied in this paper is the exoatmospheric interception of a mobile target by a thrust-controlled missile with a strap-down seeker. We aimed at defining a control law directly expressed in terms of system's measurements in a way to ensure simultaneously that the miss distance is minimized and the target remains within the field of view.

The tools used to solved the problem are issued from the field of the stabilization by output feedback for nonlinear systems. This kind of approach has received numerous attention in the field of underactuated mechanical systems (see for instance (3) and (4)). In this paper we present a way to apply passivity techniques on an interception problem.

The paper is organized as follows. We first state the equations of the exoatmospheric interception problem we have studied. We then briefly recall the

* Corresponding author : Vincent.Andrieu@gmail.com 
classical guidance and autopilot design approach. In section 4, we present a class of systems on which we can construct a stabilizing output feedback. Finally, we show in section 5 how this method can be directly applied to an exoatmospheric interception.

\section{Problem definition}

\subsection{The dynamical system}

We consider a thrust-controlled missile, and a non-propulsive target. The missile's shape is approximated as a cylinder. Let $\mathcal{R}_{M}=\left(x_{M}, y_{M}, z_{M}\right)$ in $\mathbb{R}^{3 \times 3}$ be the real matrix that characterized the reference frame associated with the missile and centered on its center of mass. Its $x_{M}$-axis is carried by the long body axis for rotational symmetry.

The correction of trajectory is done by means of a thrust system named $u_{G}$ located near the center of mass. It is produced by using four nozzles distributed around the missile, perpendicularly to its axis. Another propelling device named $u_{C}$ and located at its rear end, is used to rotate the missile around its center of mass. It consists in eight secondary nozzles positioned around the missile and making it possible to control the roll, pitch, and yaw speed.

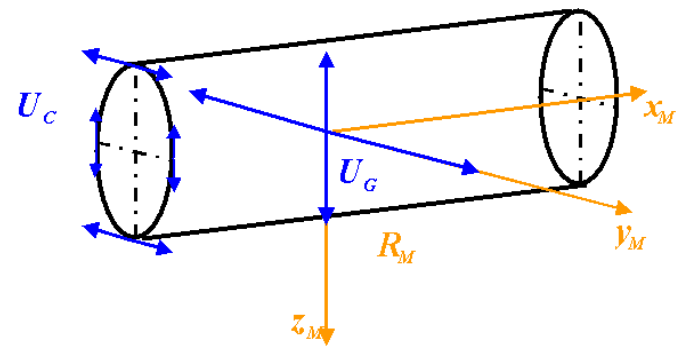

The control can be expressed in the reference frame $\mathcal{R}_{M}$ as follows :

$$
u_{G}=u_{G y} y_{M}+u_{G z} z_{M}, \quad u_{C}=u_{C x} x_{M}+u_{C y} y_{M}+u_{C z} z_{M} .
$$

The state of the system is composed of :

- $X_{r}=\left(X_{r x}, X_{r y}, X_{r z}\right)^{\top}$ in $\mathbb{R}^{3}$ denotes the coordinates of the relative position of the target.

- $V_{r}=\left(V_{r x}, V_{r y}, V_{r z}\right)^{\top}$ in $\mathbb{R}^{3}$ denotes the coordinates of the relative velocity of the target compared to the missile.

- $\mathcal{R}_{M}=\left(x_{M}, y_{M}, z_{M}\right)$ in $\mathbb{R}^{3 \times 3}$ the matrix that characterizes the attitude of the missile. 
- $\Omega=(p, q, r)^{\top}$ in $\mathbb{R}^{3}$ characterizes the rotation of the missile around its center of mass.

The gravity gradient is neglected and the mass is supposed to be constant. With these data, the system considered is :

$$
\left\{\begin{array}{rl}
\dot{X}_{r} & =V_{r} \\
\dot{V}_{r} & =\mathcal{R}_{M}^{-1}\left(\begin{array}{c}
0 \\
u_{G}
\end{array}\right)+d \\
\dot{\mathcal{R}_{M}} & =-M_{\Omega} \mathcal{R}_{M} \\
\dot{\Omega} & =u_{C}+j(\Omega)
\end{array},\right.
$$

where $j(\Omega)$ is a non linear function corresponding to the coupling products, $d$ represents the perturbations which are induced by the acceleration of the target, and $M_{\Omega}$ in $\mathbb{R}^{3 \times 3}$ is the matrix defined by :

$$
M_{\Omega}=\left(\begin{array}{ccc}
0 & -r & q \\
r & 0 & -p \\
-q & p & 0
\end{array}\right) .
$$

\subsection{The output mapping}

The sensors considered in this study are :

- Rate gyros measuring the angular speed of pitch, yaw, and roll, i.e. :

$$
\Omega=(p q r)^{\top}
$$

- An infrared strap-down seeker viewing the target and, thus, measuring :

$$
y=\left(\frac{\left(\mathcal{R}_{M} X_{r}\right)_{y}}{\left(\mathcal{R}_{M} X_{r}\right)_{x}} \frac{\left(\mathcal{R}_{M} X_{r}\right)_{z}}{\left(\mathcal{R}_{M} X_{r}\right)_{x}}\right)^{\top} .
$$

\subsection{Interception characterization}

We are considering the terminal phasis of the duel, which means that the initial values of the state satisfy : 
- The target is located forward the missile :

$$
\left\{\begin{array}{l}
\left(\mathcal{R}_{M} X_{r}\right)_{x}(0)>0 \\
\left(\mathcal{R}_{M} X_{r}\right)_{y}(0),\left(\mathcal{R}_{M} X_{r}\right)_{z}(0) \text { are small }
\end{array}\right.
$$

- The relative missile target distance decreases :

$$
V_{r}(0)^{\top} X_{r}(0)<0
$$

The interception problem consists in finding a control law such that the miss distance goes to zero under constraints such as saturation on acceleration level and limitation of field of view.

In order to determine such a law, we define a function $\phi$ depending on the entire state that characterizes the interception configuration. Specifically, $\phi$ should be such that all solutions of the system (1) which remain in the set :

$$
\left\{\left(X_{r}, V_{r}, \mathcal{R}_{M}, \Omega\right): \phi\left(X_{r}, V_{r}, \mathcal{R}_{M}, \Omega\right)=0\right\}
$$

lead to interception. Choice of $\phi$ could be for instance the predicted miss distance or the angular difference between the actual configuration and collision triangle, the cross product of the relative speed vector and the vector missile / target. After selecting the function $\phi$, it remains to determine the control law that regulates $\phi$ around the origin, despite perturbations on the system.

\section{Classical method}

In this section we briefly recall the classical method used to construct a flight control law solution of the interception problem. The reader could refer to (6) or (2) for further details.

\subsection{State feedback design}

The classical approach for solving interception problem consists in defining, in a decoupled way, a guidance law and then an autopilot that realizes the acceleration orders adequately. Both guidance and autopilot require the knowledge of the entire state of the system.

This procedure arises from considering that the missile rotation with respect 
to its center of mass evolves faster than its translation movement:

$$
|\overbrace{\left(\begin{array}{c}
\mathcal{R}_{M} \\
\Omega
\end{array}\right)}^{\cdot}|>|\overbrace{\left(\begin{array}{c}
X_{r} \\
V_{r}
\end{array}\right)}^{\cdot}|
$$

Frequential decoupling of the system (1) in terms of guidance and autopilot controls is then possible.

\subsubsection{Guidance Law}

The state system associated with guidance problem is :

$$
\left\{\begin{array}{l}
\dot{X}_{r}=V_{r} \\
\dot{V}_{r}=\Gamma_{M}+d
\end{array}\right.
$$

The control input $\Gamma_{M}$ is the required acceleration of the center of mass.

The objective is to find a control law $G$ such that, if $\Gamma_{M}=G\left(X_{r}, V_{r}\right)$, then, along the trajectories of the guidance system $(2), \phi\left(X_{r}, V_{r}\right)$ is regulated around zero. Optimal control theory (see (6)), is used to solve this problem. Constraints on the acceleration level and direction should be taken into account.

\subsubsection{Flight Control System}

The autopilot (or flight control) loop aims at orientating the missile so that the acceleration of the center of mass $\Gamma_{M}$ determined by the guidance subsystem can be realized. The autopilot control design uses the system representation :

$$
\left\{\begin{aligned}
\mathcal{R}_{M} & =-\Omega \mathcal{R}_{M} \\
\dot{\Omega} & =u_{C}+j(\Omega) .
\end{aligned}\right.
$$

The control law $\left(u_{G}, u_{C}\right)=C\left(\mathcal{R}_{M}, \Omega, X_{r}, V_{r}\right)$ is defined to insure that the resulting acceleration $\Gamma_{M}=\mathcal{R}_{M}^{-1}\left(\begin{array}{c}0 \\ u_{G}\end{array}\right)$ is regulated around the input $G\left(X_{r}, V_{r}\right)$ given by the guidance law.

The missile rotation :

$$
\mathcal{R}_{M} \Gamma_{M}=\left(\begin{array}{c}
0 \\
u_{G}
\end{array}\right) \Rightarrow \mathcal{R}_{M} \text { has to satisfy }\left(\mathcal{R}_{M} \Gamma_{M}\right)_{x}=0,
$$


A first order approximation of the flight control system (3) can be used to determine the autopilot law.

\subsection{Control implementation}

The two-step design we have described provides the control law $\left(u_{G}, u_{C}\right)=$ $C\left(\mathcal{R}_{M}, \Omega, X_{r}, V_{r}\right)$. Its implementation requires reconstruction of the system's state from the available measurements. The resulting state estimate is used in the state feedback law.

\subsection{Disadvantages of classical design technique}

Despite that this method has been used successfully for a long time, it presents some drawbacks. The non-linearity of the system is generally not taken into account to build the control law. Linearized models are more commonly used which limits the validity of the controlled system to a neighborood of the linearization point.

Furthermore, the use of state estimates in the control law increases the global time response of the controlled system and may produce other perturbations.

We shall now present a method, based on an output feedback design, and apply it to the (simpler) case of exoatmospheric interception. The control law will directly be expressed as a function of the measurements, and linearization of the system considered is unnecessary.

\section{Output feedback for a specific class of system}

In this subsection, let first introduce a theorem which states, for a special class of systems, the existence of a dynamic output feedback which regulates a state function $\mathcal{V}$ around the origin.

Theorem 1 We consider a dynamical system on $\mathbb{R}^{n}$ and its observation mapping in $\mathbb{R}^{m}$ :

$$
\dot{x}=f(x, u), \quad y=h(x) .
$$

where $u$ the control is in $\mathbb{R}^{p}$. Let $\mathcal{V}$ be a function of the state $x$ taking values in $\mathbb{R}^{+}$. Suppose there exists real functions $\chi, \eta$ and $\beta$ from $\mathbb{R}^{m}$ in $\mathbb{R}^{p}$ such that we have :

- For each $x$ in $\mathbb{R}^{n}$ and $u$ in $\mathbb{R}^{p}$, we have : 


$$
\frac{\partial \mathcal{V}}{\partial x}(x) f(x, u) \leq\left(\frac{\partial \eta(y)}{\partial x} f(x, u)+\beta(y)\right)^{\top}(u-\chi(y))
$$

- We have the inclusion ${ }^{1}$ :

$$
\left\{x(t): \frac{\partial \eta(y)}{\partial x} f(x, \chi(y))+\beta(y) \equiv 0\right\} \subseteq\{x(t): \mathcal{V}(x) \equiv 0\}
$$

Then, there exists a dynamic output feedback such that for each $x(t)$ solution of the closed system, which is bounded in positive time we have:

$$
\lim _{t \rightarrow+\infty} \mathcal{V}(x(t))=0
$$

Proof : For this special class of system an output feedback method has been formalized by a mini-max approach by Prieur and Praly in (8). Following their approach, we introduce the dynamic output feedback with state $w$ :

$$
\left\{\begin{array}{l}
\dot{w}=\psi(w, y) \\
u=\theta(w, y)
\end{array}\right.
$$

and the function :

$$
\mathcal{W}(x, w)=\mathcal{V}(x)+\frac{1}{2}(w-\eta(y))^{\top}(w-\eta(y)) .
$$

From (5), the time derivative of $\mathcal{W}$ along the trajectories of the system (1) under the feedback (7), satisfies :

$$
\begin{aligned}
& \overparen{\mathcal{W}(x, w)} \leq \underbrace{(\dot{\eta(y)}+\beta(y))^{\top}(\theta(w, y)-\chi(y)-w+\eta(y))}_{\theta(w, y)=\chi(y)+w-\eta(y) \text { makes this term equal zero }} \\
& +\underbrace{(\psi(w, y)+\beta(y))^{\top}(w-\eta(y))}_{\psi(w, y)=\eta(y)-w-\beta(y) \text { makes this term negative }} .
\end{aligned}
$$

The dynamic output feedback (7) is then ;

$$
\left\{\begin{array}{l}
\dot{w}=\eta(y)-w-\beta(y) \\
u=\chi(y)+w-\eta(y)
\end{array}\right.
$$

which gives :

$$
\begin{aligned}
\overparen{\mathcal{W}(x, w)} & \leq-(w-\eta(y))^{\top}(w-\eta(y)) \\
& \leq 0
\end{aligned}
$$

$\overline{1}$ the notation $m(x) \equiv n(x)$ means $m(x(t))=n(x(t))$ for all $t$ where $x(t)$ is a trajectory of the system (4). 
From LaSalle Invariance Principle, (see (5, theorem X.1.3)) it follows that each bounded solution $(x(t), w(t))$ converges to the set :

$$
\{(x(t), w(t)): w \equiv \eta(y)\} .
$$

And, with (8) :

$$
\{(x(t), w(t)): w \equiv \eta(y)\} \subseteq\{(x(t), w(t)): u \equiv \chi(y), \stackrel{\cdot \dot{\eta(y)}}{\equiv} \equiv-\beta(y)\}
$$

Consequently, we get :

$$
\lim _{t \rightarrow+\infty} x(t) \in\left\{x(t): \frac{\partial \eta(y)}{\partial x} f(x, \chi(y))+\beta(y) \equiv 0\right\}
$$

and with (6) :

$$
\lim _{t \rightarrow \infty} \mathcal{V}(x(t))=0
$$

Remarks :

This method is a simple extension of well-known results in the framework of Euler-Lagrange systems. It has been first implemented for robotics systems (see $(7))$.

\section{Application to the exoatmospheric interception}

First of all, in order to simplify the system representation we introduce new state coordinates :

$$
X=\mathcal{R}_{M} X_{r}, \quad V=\mathcal{R}_{M} V_{r} .
$$

Using this set of coordinates, the system becomes :

$$
\left\{\begin{aligned}
\dot{X} & =V+X \wedge \Omega, \\
\dot{V} & =\left(\begin{array}{c}
0 \\
u_{G}
\end{array}\right)+V \wedge \Omega, \\
\dot{\mathcal{R}_{M}} & =-M_{\Omega} \mathcal{R}_{M}, \\
\dot{\Omega} & =u_{C}+j(\Omega),
\end{aligned}\right.
$$

where $d$, the target acceleration, is neglected. Furthermore, the output mapping becomes :

$$
\left\{\begin{array}{l}
y=\left(\frac{X_{y}}{X_{x}} \frac{X_{z}}{X_{x}}\right), \\
\Omega .
\end{array}\right.
$$


The criterion function $\phi$ that characterizes the results of the interception is chosen as the cross product of the relative speed vector and the vector missile / target. As the seeker is strap-down, we also want the relative position of the target to remain close to the x-axis, which means that we want $X_{y}$ and $X_{z}$ to remain as small as possible. In order to insure good quality of the seeker measurement, we aim at stabilizing the vector $\Omega$ around the origin. Consequently, the criterion $\phi$ that fully characterizes the expected performances of the interception is defined by :

$$
\phi=\left(\begin{array}{l}
\phi_{1} \\
\phi_{2} \\
\phi_{3}
\end{array}\right), \text { with } \quad \phi_{1}=X \wedge V, \quad \phi_{2}=\left(\begin{array}{c}
X_{y} \\
X_{z}
\end{array}\right), \quad \phi_{3}=\Omega .
$$

In order to find a control law which regulates $\phi$ around the origin, we introduce a positive state function $\mathcal{V}$ associated to the criterion $\phi$ defined by :

$$
\mathcal{V}=\frac{1}{2}\left(\phi_{1}^{\top} \phi_{1}+X_{x} \phi_{2}^{\top} \phi_{2}+X_{x}^{3} \Omega^{\top} \Omega\right)
$$

Due to the fact that we suppose $X_{x}$ always strictly positive, $\mathcal{V}$ satisfies :

$$
\mathcal{V}=0 \Rightarrow \phi=0
$$

and, consequently, the problem is now to regulate $\mathcal{V}$ around the origin. This will be done by using the same procedure that in the previous section.

By differentiating $\mathcal{V}$ along the system (9), we obtain :

$$
\begin{aligned}
\dot{\mathcal{V}}= & X_{x}^{3} \dot{y}^{\top}\left(m(y) u_{G}+y\right) \\
& +X_{x}^{3} \Omega^{\top}\left(l(y) m(y) u_{G}+n(y) y^{\top} y+u_{C}+j(\Omega)\right) \\
& +\frac{3}{2} V_{x} X_{x}^{2}\left(y^{\top} y+\Omega^{\top} \Omega\right) .
\end{aligned}
$$

where $l: \mathbb{R}^{2} \rightarrow \mathbb{R}^{3 \times 2}, m: \mathbb{R}^{2} \rightarrow \mathbb{R}^{2 \times 2}$, and $n: \mathbb{R}^{2} \rightarrow \mathbb{R}^{2}$ are defined as :

$$
l(y)=\left(\begin{array}{cc}
-y_{z} & y_{y} \\
-y_{y} y_{z} & -1-y_{z}^{2} \\
1+y_{y}^{2} & y_{y} y_{z}
\end{array}\right), \quad m(y)=\left(\begin{array}{cc}
1+y_{z}^{2}-y_{y} y_{z} \\
y_{y} y_{z} & 1+y_{y}^{2}
\end{array}\right), \quad n(y)=\frac{3}{2}\left(\begin{array}{c}
0 \\
-y_{z} \\
y_{y}
\end{array}\right)
$$

So following Section 4 we extend $\mathcal{V}$ as :

$$
\mathcal{W}=\mathcal{V}+\frac{X_{x}^{3}}{2}(w-y)^{\top}(w-y)
$$


By differentiating $\mathcal{W}$ along the trajectories of the system we obtain :

$$
\begin{aligned}
\dot{\mathcal{W}}= & X_{x}^{3} \dot{y}^{\top}\left(m(y) u_{G}+2 y-w\right) \\
& +X_{x}^{3} \dot{w}^{\top}(w-y) \\
& +X_{x}^{3} \Omega^{\top}\left(u_{C}+j(\Omega)+n(y) k(\Omega, y, w)+l(y) m(y) u_{G}\right) \\
& +\frac{3}{2} V_{x} X_{x}^{2} k(\Omega, y, w)
\end{aligned}
$$

where $k$ is the positive function defined by :

$$
k(a, y, w)=y^{\top} y+\Omega^{\top} \Omega+(w-y)^{\top}(w-y) .
$$

As the matrix $m(y)$ is invertible, discarding the terms in $\dot{y}$ is performed by choosing :

$$
u_{G}=m(y)^{-1}(w-2 y)
$$

and by taking :

$$
\begin{aligned}
\dot{w} & =-w+y \\
u_{C} & =-\Omega-j(\Omega)-n(y) k(\Omega, y, w)-l(y) m(y) u_{G} .
\end{aligned}
$$

the time derivative of $\mathcal{W}$ becomes :

$$
\dot{\mathcal{W}}=X_{x}^{3}\left(-|w-y|^{2}-|\Omega|^{2}\right)+\frac{3}{2} V_{x} X_{x}^{2} k(\Omega, y, w) .
$$

and the stabilization of $\phi$ around the origin is deduced from the LaSalle invariance principle and the fact that $V_{x}<0$.

The main disadvantage of this method is that the convergence of the criterion around the origin is asymptotic. The interception problem is a finite time problem. In practice, the control has to correct the trajectory of the missile in a short time. The convergence can be accelerated by adding gains in the expression of the control law.

\section{Simulation}

The stability and performances of the controller has been tested in simulations of exoatmospheric interception. The example below illustrates the behaviour of the controlled system. 


\subsection{Initial state}

The initial values of the relative position and speed are :

$$
X=\left(\begin{array}{c}
5000 \\
-410 \\
-400
\end{array}\right), \quad V=\left(\begin{array}{c}
-300 \\
5 \\
-5
\end{array}\right)
$$

The initial miss distance is $400 \mathrm{~m}$ that has to driven to zero by the control law. Furthermore, we suppose the initial $\Omega$ vector to be different from zero :

$$
\Omega=\left(\begin{array}{c}
0.01 \\
-0.01 \\
0.01
\end{array}\right)
$$

Even if the control law is computed for perfect conditions, several perturbations have been considered in the simulated model :

- the mass of the missile decreases according to consumption,

- the center of gravity is not located on the symmetry axis and evolves during the duel,

- saturations on thrust controls are taken into account and the thrust response of the engines have been modelled,

- measurements are corrupted by additive noise,

- target is submitted to random accelerations corresponding to non-uniform repartition of mass.

\subsection{Results}

By using the control law developed in the previous chapter, the miss distance obtained in presence of noise measurement and saturation is about $20 \mathrm{~cm}$. As we can see on the graphics, the criterion is regulated around zero in nearly 7 seconds. After this delay, the target is exactly forward the missile and the interception is guaranteed. 

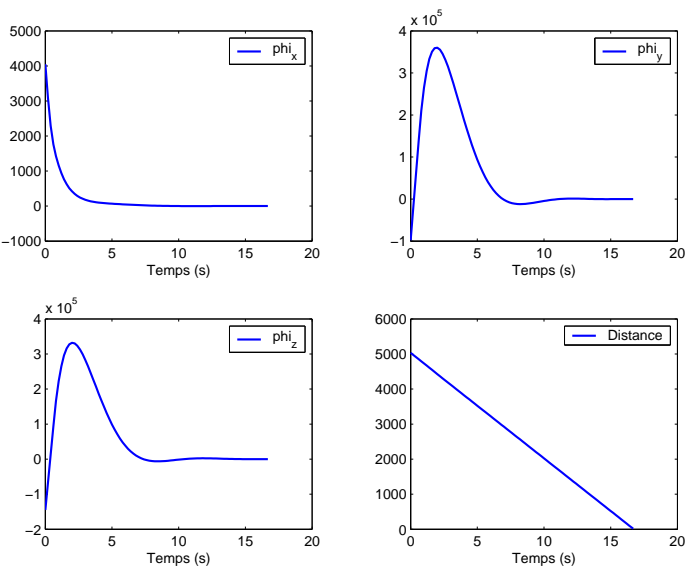

Figure 1. $\phi_{1}$ evolution

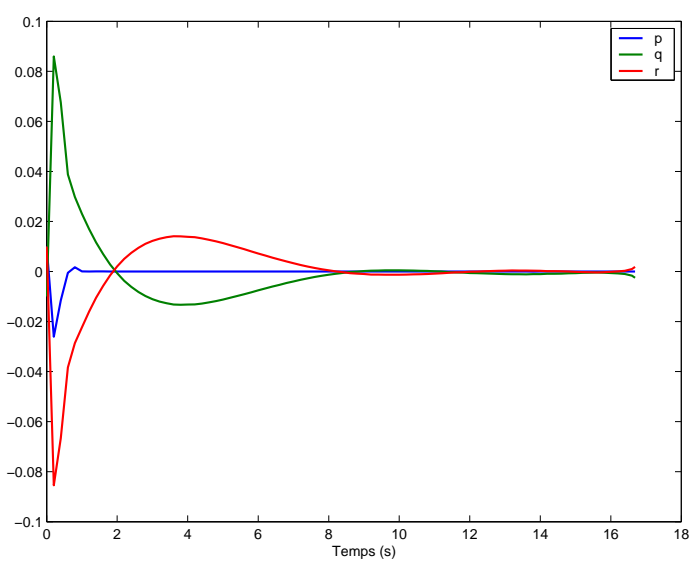

Figure 2. $p, q, r$ evolution

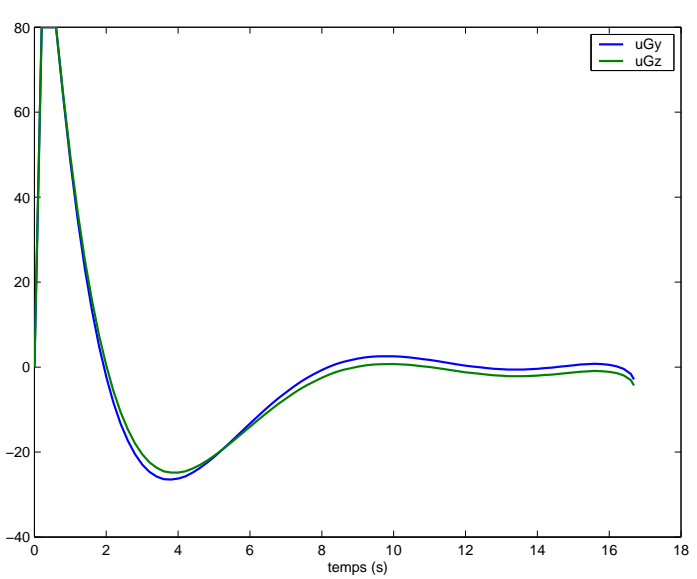

Figure 3. $U_{g}$ control law

\section{Conclusion and perspectives}

We have investigated the possibility of applying non-linear control design to interception problems. More specifically, we have shown that a direct output 


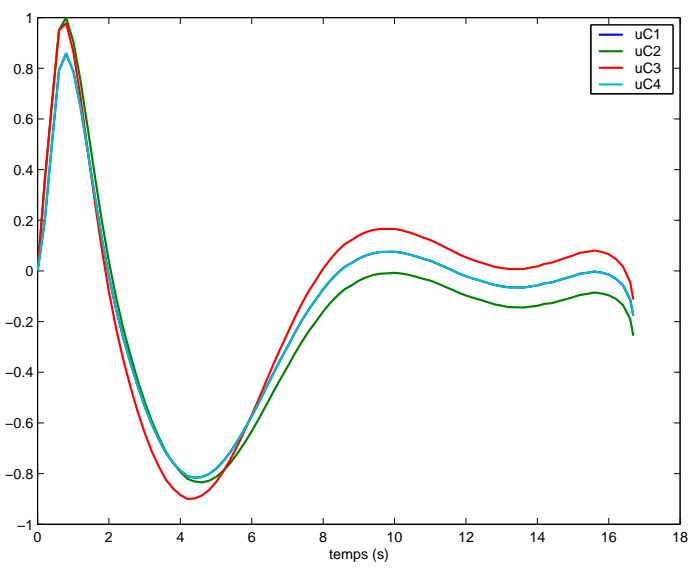

Figure 4. $U_{c}$ control law

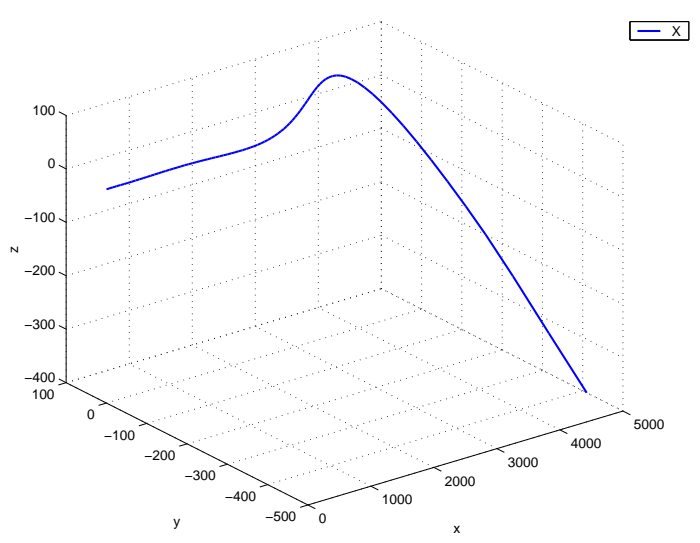

Figure 5. Relative position of the target in $\mathcal{R}_{M}$

feedback design can be used to solve the problem of exoatmospheric interception with a non-manoeuvring target. Extension of this approach to endoatmospheric duel is currently under investigation using the work of Akmeliawati and Mareels in (1).

\section{Acknowledgements}

The authors would like to thank Dr. Laurent Praly for his helpful comments.

\section{References}

[1] R. Akmeliawati, I. Mareels, Nonlinear Energy-based Control Method for Aircraft Dynamics, Proc. of the 40th IEEE CDC, Orlando, (2001) .

[2] R. Carpentier, Guidage des avions et des missiles aerodynamiques, Cours SupAERO, (1984). 
[3] K.D. Do, Z.P. Jiang, J. Pan and H. Nijmeijer, A global output-feedback controller for stabilization and tracking of underactuated ODIN: a spherical underwater vehicle, Automatica, vol. 40, no. 1, pp. 117-124, (2004).

[4] K. D. Do, Z. P. Jiang and J. Pan, On global tracking control of a VTOL aircraft without velocity measurements, IEEE Trans. on Automatic Control, vol. 48, no. 12, pp. 2212-2217, (2003).

[5] J. K. Hale, Ordinary Differential Equations, Third Edition, Krieger Pub. Comp., (1980).

[6] C.-F. Lin, Modern Navigation, Guidance, and Control Processing, Vol. II , Prentice Hall, Englewood Cliffs, New Jersey, (1991).

[7] R. Ortega, A. Loria, P. J. Nicklasson and H. Sira-Ramirez, Passivity-based control of Euler-Lagrange systems, Springer-Verlag, Berlin, Communications and Control Engineering, Sept. (1998).

[8] C. Prieur, L. Praly, A tentative direct output Lyapunov design of output feedback, Proc. of the 6th IFAC Symposium on Nonlinear Control Systems. September (2004). 